\title{
Conical Electron Tomography of a Chemical Synapse: Polyhedral Cages Dock Vesicles to the Active Zone
}

\author{
Guido A. Zampighi, ${ }^{1,2}$ Nick Fain, ${ }^{1}$ Lorenzo M. Zampighi, ${ }^{3}$ Francesca Cantele, ${ }^{4}$ Salvatore Lanzavecchia, ${ }^{4}$ and \\ Ernest M. Wright ${ }^{3}$ \\ ${ }^{1}$ Department of Neurobiology, ${ }^{2}$ Jules Stein Eye Research Institute, and ${ }^{3}$ Department of Physiology, University of California, Los Angeles School of Medicine, \\ Los Angeles, California 90095, and ${ }^{4}$ Department of Structural Chemistry, School of Pharmacy, University of Milan, 20122 Milan, Italy
}

In this study, we tested the hypothesis that the structure of the active zone of chemical synapses has remained uncertain because of limitations of conventional electron microscopy. To resolve these limitations, we reconstructed chemical synapses of rat neocortex, the archetypical "average" synapse, by conical electron tomography, a method that exhibits an isotropic in plane resolution of $\sim 3 \mathrm{~nm}$ and eliminates the need to impose symmetry or use averaging methods to increase signal-to-noise ratios. Analysis of 17 reconstructions by semiautomatic density segmentation indicated that the active zone was constructed of a variable number of distinct "synaptic units" comprising a polyhedral cage and a corona of approximately seven vesicles. The polyhedral cages measured $\sim 60 \mathrm{~nm}$ in diameter, with a density of $\sim 44 / \mu \mathrm{m}^{2}$ and were associated with vesicles at the active zone ("first tier"). Vesicles in this first-tier position represented $\sim 7.5 \%$ of the total number of vesicles in the terminal and were contiguous, hemifused ( $\sim 4 \%$ of total), or fully fused ( $\sim 0.5 \%$ of total) to the plasma membrane. Our study supports the hypothesis that rat neocortical synapses are constructed of variable numbers of distinct synaptic units that facilitate the docking of vesicles to the active zone and determine the number of vesicles available for immediate release.

Key words: chemical synapses; synaptic vesicles; vesicular pools; docking; hemifusion; cytomatrix

\section{Introduction}

A key feature of synaptic transmission is that vesicle fusion and release of neurotransmitters occurs only at discrete regions of the presynaptic neurons, called the "active zone" (Couteaux and Pecot-Dechavassine, 1970). In the electron microscope, clusters of synaptic vesicles contained within a distinct protein matrix, called the "cytomatrix," constitute the active zone (Landis et al., 1988; Hirokawa et al., 1989; Zhai and Bellen, 2004). Previous studies indicate that the structure of the cytomatrix depends on the type of synapse and animal species under study. In mammalian central synapses, $\sim 60 \mathrm{~nm}$ diameter "dense" particles that self-assemble into arrays, called the "presynaptic grid," comprise the cytomatrix (Gray, 1963; Ackert et al., 1972; Pfenninger et al., 1972). In neuromuscular junctions of frog, it appears as an arrangements of "ribs," "beams," and "pegs" are referred to as the "active zone material" (Harlow et al., 2001), whereas in neuromuscular junctions of Drosophila, the active zone consists of "T bars" (Meinerzhagen, 1996; Meinerzhagen et al., 1998). In "ribbon" synapses of rat and mouse retina, the cytomatrix appears as plates, whereas in saccular hair cells of frogs, the structure is more

Received 0ct. 11, 2007; revised March 10, 2008; accepted March 11, 2008.

This work was supported by National Institutes of Health (NIH) Grant EY-04110 and the 0ppenheimer Foundation (G.A.Z.), FIRST 2001 (S.L.), and NIH Grant DK44602 (E.M.W.).

Correspondence should be addressed to Guido A. Zampighi, Department of Neurobiology, University of California, Los Angeles School of Medicine, Los Angeles, CA 90095. E-mail: gzampighi@mednet.ucla.edu.

DOI:10.1523/JNEUROSCI.4639-07.2008

Copyright $\odot 2008$ Society for Neuroscience $\quad$ 0270-6474/08/284151-10\$15.00/0 that of dense spheres (Lenzi et al., 1999; von Gersdorff, 2001; Dick et al., 2003).

Despite the remarkable structural heterogeneity, the cytomatrix in all these synapses is thought to enable docking and fusion of vesicles to the active zone. This hypothesis originated with Gray' seminal studies showing that dense particles link vesicles to depressions in the active zone, called synaptopores (Ackert et al., 1972; Pfenninger et al., 1972), the dimensions of which increase on electrical stimulation (Triller and Korn, 1985). Additional studies supporting this hypothesis include the observation that the emergence of these dense particles correlates precisely with the maturation of central synapses (Bloom and Aghajanian, 1966) and that their chemical composition involves a myriad of proteins involved in cell adhesion, membrane fusion, and endocytosis (Phillips et al., 2001).

Despite this extensive body of experimental evidence, the hypothesis that dense particles facilitate vesicle docking and fusion remains unconventional. Presynaptic grids are thought to embody a specialization that, although present in some synapses, does not reflect an inherent property of "average" synapses. Consequently, current models explaining synaptic transmission view vesicle docking as a multistep, highly regulated process involving the assembly of complexes between proteins in the membrane of the vesicle (synaptobrevin) with proteins in the plasma membrane [syntaxin, SNAP-25 (synaptosome-associated protein of $25 \mathrm{kDa}$ ) ] (Jahn and Scheller, 2006). Working in conjunction with SNAP receptors (SNAREs), other protein complexes have also been proposed to participate in docking, priming, and the teth- 
ering of vesicles to the active zone (for review, see Zhen and Jin, 2004). The lack of structural information, however, has precluded establishing the possible relationship between these complexes and the dense particles first identified by Gray in central synapses.

In this study, we tested the possibility that, although presynaptic grids might be singular structures, dense particles are intrinsic structures of central synapses. We speculate that their presence might have been obscured by the limitations of conventional electron microscopy, including the "projection artifact," a result of projecting the entire thickness of the section onto a single plane. To resolve these limitations, we reconstructed chemical synapses of rat neocortex, the archetypical average synapse, by conical electron tomography and analyzed the maps using semiautomatic density segmentation (Lanzavecchia et al., 2005; Zampighi et al., 2005; Cantele et al., 2007; Salvi et al., 2008). At $\sim 3 \mathrm{~nm}$, the resolution of these methods is sufficiently high to identify vesicles that are hemifused with the membrane of the active zone (Zampighi et al., 2006).

Analysis of 17 reconstructions indicated that the active zone was constructed of a variable number of "units" comprising a polyhedral cage and a corona of vesicles. Our study strongly supports the hypothesis that rat neocortical synapses are constructed of variable numbers of these "synaptic units" that facilitate the binding and docking of vesicles to the active zone.

\section{Materials and Methods}

The steps used to prepare the conical tomograms are listed in Table 1. The following paragraphs describe each step in an abbreviated form because they have been described in detail previously (Zampighi et al., 1980, 1989, 1997, 2005; Lanzavecchia et al., 2005; Salvi et al., 2008)

Specimen preparation. Tissue from the frontal association and motor neocortices was dissected from the brains of Sprague Dawley adult rats. Brains were perfused with aldehydes, postfixed in $\mathrm{OsO}_{4}$, dehydrated in ethanol, and embedded in Epon 812 (Zampighi et al., 1980, 1989, 1997). Thin sections of thickness with gray-to-silver interference color (55-80 $\mathrm{nm}$ ) were cut and deposited on 200 mesh grids coated with carbon or single-hole ( $\sim 600 \mu \mathrm{m}$ diameter) grids coated with Formvar and carbon. The use of single-hole grids eliminated the interference that bars introduce when the specimen was tilted and rotated. The grids were floated on solutions of uranyl and lead, and their surfaces were peppered with 10$\mathrm{nm}$-diameter gold particles. The particles served as fiduciary markers for centering and preliminary alignment of the conical series. Thin layers of carbon were deposited on the exposed surfaces (triple carbon layer) to decrease the deformation ("shrinkage") induced by radiation damage.

In an effort to show that the preparative methods used for the neocortices produce reliable results, we applied them to solutions of two "known" proteins (F-actin and anti-actin IgG). The resulting reconstructions fit models of these proteins calculated by $x$-ray diffraction methods at higher resolution. Images of these reconstructions are included in the supplemental material (available at www.jneurosci.org as supplemental material).

Collection of conical series, centering, alignment, and preliminary reconstruction. We collected 17 conical series of images in the Gatan (Pleasanton, CA) 650 Single Tilt Rotating Holder in an FEI (Hillsboro, OR) Tecnai 12 electron microscope operated at $120 \mathrm{kV}$. The images were collected in a $2048 \times 2048$ CCD Gatan camera at 34,230× final magnification (pixel size, $0.409 \mathrm{~nm}$ ). Imaging was performed using a minimum-dose method: searching was done at $2700 \times$ magnification with minimum illumination, and the regions of interest were imaged by focusing $\sim 2 \mu \mathrm{m}$ away. The thin sections were tilted $\left(55^{\circ}\right.$ or $\left.60^{\circ}\right)$ and rotated in $3.5^{\circ}$ or $5^{\circ}$ steps through a complete $360^{\circ}$ turn. The decrease in the relative distance between gold particles in untilted projections collected before and after completing the series estimated the shrinkage induced by radiation damage.

Manual prealignment involved using the NIH ImageJ software pack-
Table 1. The steps used to prepare the conical tomograms

Preparation of Specimens: Plastic Embedded Sections<smiles>[CH]C=C</smiles>

\section{Collection of Conical Series}<smiles>c1ccccc1</smiles>

Alignment Based on Fiduciary Markers<smiles>[CH]=C</smiles>

Preliminary Reconstruction

$\sqrt{3}$

"Global" and "Local" Refinement

$\sqrt{ }$

Final Reconstruction<smiles>[CH]=C</smiles>

Estimate of Resolution<smiles>[CH]=C</smiles>

Visualization and Density Segmentation

age (Abramoff et al., 2004) to select a specific gold particle and shifting each image of the conical series such that the particle was in the center of all projections. After centering, the coordinates of other gold particles (five to eight) allowed us to find the orientation parameters (Euler angles and origin position) of the micrographs and calculate "preliminary" three-dimensional (3D) maps with the weighted back-projection algorithm (Radermacher, 1992).

"Global" and "local" refinement. The preliminary maps were refined first by using a "global" strategy in which the projections were iteratively cross-correlated with reprojections of an updated reconstruction (i.e., projection matching). During the computation of the reprojections, we tested incremental adjustments in the three projection parameters, $(\alpha, \beta$, and $\gamma$ ) and monitored the results by statistical indices based on the variance of the volume (Zampighi et al., 2005). This global refinement strategy allowed the determination of the best values for all the shift and orientation parameters and improved both alignment and resolution of the preliminary maps.

Because the deformation induced by radiation damage (shrinkage) was nonlinear, the globally refined maps were further refined with a "local" refinement strategy (Cantele et al., 2007). The basic steps of the strategy involved the following: (1) partition of the preliminary reconstruction into subvolumes; (2) extraction of corresponding subareas for each subvolume from the micrographs of the tilt series; (3) reprojection of each subvolume according to the orientation parameters; and (4) refinement of these parameters by correlating each subarea to the corresponding computed projection. In this manner, the underlying strategy of projection matching as it applies to tomography (Zampighi et al., 2005) was combined with the ability to compute complete reconstructions by pasting together independently reconstructed subvolumes (Lanzavecchia et al., 2005). 


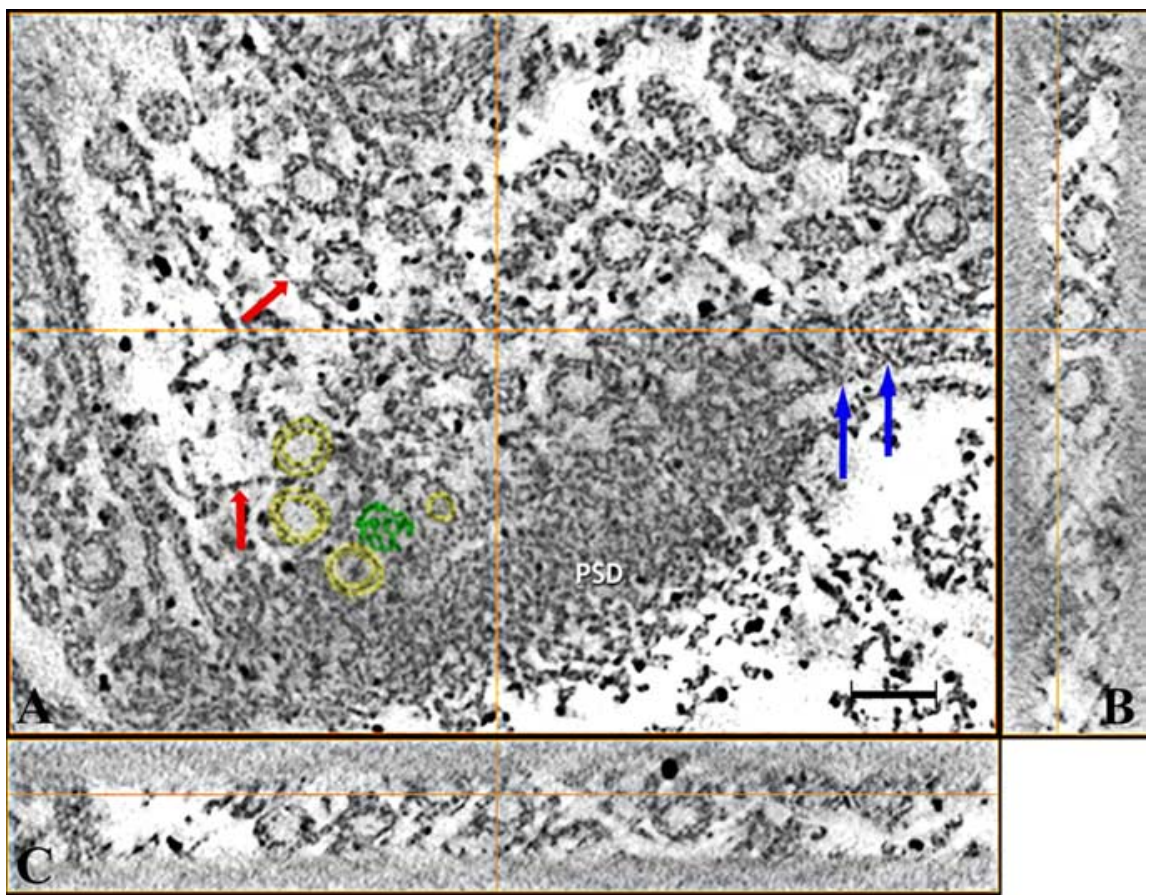

Figure 1. Planes of a reconstructed synapse. $\boldsymbol{A}$ shows a plane along the $x-y$ direction to demonstrate the overall distribution of synaptic organelles. The presynaptic neuron contains clusters of vesicles that appear as circles bound by the trilayer structure representing the phospholipid bilayer of the membrane. The active zone, the region in which vesicles dock and fuse, faces a thick layer of density associated to the PSD. The blue arrows point to two vesicles at the edge of the active zone and in close proximity to the plasma membrane. One of them is hemifused, abutting another syndesome (not resolved in this plane of the reconstruction). The syndesome appears as a cage associated to the active zone (green) and surrounded by a partial corona of synaptic vesicles (yellow). Long filaments associate to vesicles in the cytoplasm of the terminal (red arrows). The intersecting brown lines indicate the location where planes along the $z$-direction were sectioned in the reconstructed volume. $B$ and $C$ show the $x-z$ and $y-z$ planes, respectively. The synaptic vesicles appear as circles bound by the unit membrane pattern representing the phospholipids bilayer of the membrane. Because the overall thickness of this pattern measured $\sim 6 \mathrm{~nm}$, it follows that the resolution of the map, at one-half this thickness, would be $\sim 3 \mathrm{~nm}$. Note that the resolution of the map extends equally well in all directions. Scale bar, $75 \mathrm{~nm}$.

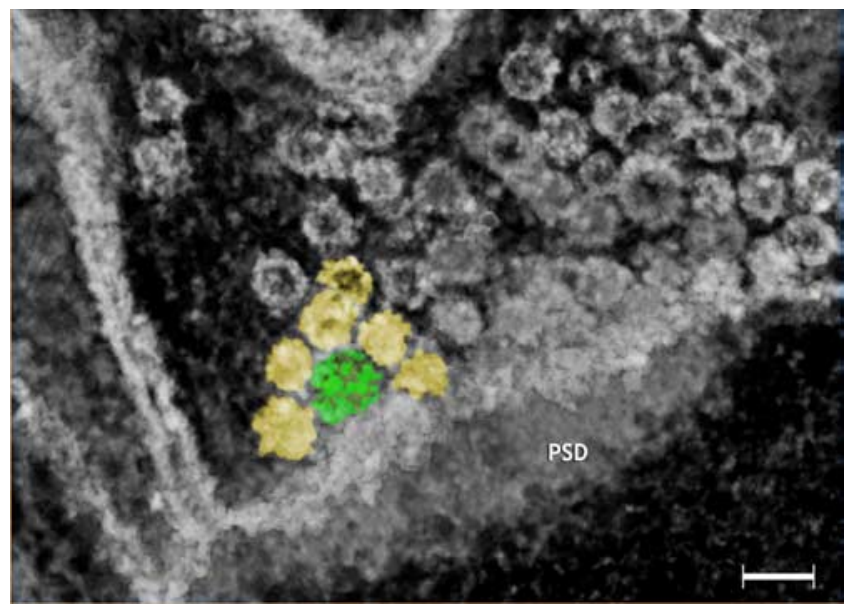

Figure 2. The rendered volume of the reconstructed synapse. Visualization of the 3D structure of the synapse by volume rendering involves assigning light intensities to each voxel of the reconstructed volume. Rendering requires inverting the contrast of the planes so that matter is white and the lack thereof is black. Volume rendering shows vesicles as spheres instead of circles bounded by the unit membrane pattern (yellow). The plasma membranes appear as wavy surfaces (white bands) and the syndesome as a cage instead of a dense particle (green). Vesicles at the active zone are arranged as a corona (yellow) around the syndesome. The cytomatrix appears as ubiquitous densities, which are associated to the plasma membranes and which occupy the cytoplasm of the terminal. The thick layer of density located underneath the plasma membrane corresponds to the PSD. Scale bar, $75 \mathrm{~nm}$.
The local refinement strategy also yielded a $3 \mathrm{D}$ volume and a set of orientation parameters for the $N$ projections: $\left\{\alpha_{i}, \beta_{i}, \gamma_{i}, \Delta x_{i}, \Delta y_{i} i=\right.$ $0,1, \ldots N\}$. The reconstructed "slab" volume was then divided into $M$ small cubes, for which one edge of each corresponds to the height of the slab: $C_{j}, j=0,1, \ldots M$. Each cube was then reconstructed from subregions of the properly extracted original micrographs. To extract these corresponding areas $(256 \times 256$ pixels $)$, we projected the center of each cube using the orientation parameters that obtained with the preliminary alignment process. Stacks of these small areas together with the Euler angles from the original map reconstructed the cubes. Because the projections were already centered, the shift parameters were set to 0 , and the size of this new reconstruction increased to $256 \times 256 \times 128$ for reliable reprojections.

The orientation parameters of each cube were then refined, as was done for the entire reconstruction, and the resulting reconstructions were projected again, along the directions dictated by the Euler angles. The contribution of the projection corresponding to the chosen viewing direction was subtracted from the volume to avoid bias in the subsequent projection matching. To determine a new shift value, the computed projections were correlated with the experimental projections extracted from the micrograph. The 64 cubes created in this process were reconstructed a second time varying the shift parameters but leaving the Euler angles constant. Calculation of the final reconstruction of the synapse required 64 parameter files rather than the single file required when applying the global refinement strategy.

Visualization of the 3D maps and density segmentation. We used the Amira software package to visualize reconstructed $3 \mathrm{D}$ sections of synapses, using volume-rendering techniques, which involve assigning colors and intensities of light to each voxel based on a number of userdefined parameters. To facilitate studying the relationships between different organelles, we segmented the maps on the basis of density thresholds and/or topological considerations. Segmentation was performed both manually, by using tools of the Amira package, and semiautomatically by using JUST (Salvi et al., 2008). The semiautomatic segmentation process involved the following: (1) creation of a 3D watershed map (Vincent and Soille, 1991) of the volume; (2) extraction of the background noise; (3) extraction of vesicles, plasma membranes, gold particles, and syndesomes; (4) extraction of regions with high, medium, and low densities, regions that represented no "known" organelles but fit within a certain density range; and (5) composition of a final segmentation map in which all segmented structures were analyzed and conflicting assignments were resolved.

The segmented maps were volumes with the same size and topology of the original. In the segmented map, however, each voxel was an integer number assigned with an order that is fixed during the composition step. Information about density variation was recovered by combining the segmentation map $S$ with the original volume $V$. For each voxel, $v \in V$, the corresponding value $s \in S$ was tested for a specific label $s^{\prime}\left(s==s^{\prime}\right)$. A simple operation of logical and between $V$ and the tested version of $S$ produces a new volume $V^{\prime}=V$ and $\left(S==s^{\prime}\right)$. In $V^{\prime}$, all voxels are zeroed apart from those belonging to the object associated to the label $s^{\prime}$, which maintains their true density values. Instead of a single label $s^{\prime}$, an interval $\left[s^{\prime}, s^{\prime \prime}\right]$ can also be used. The volume $V^{\prime}$ was visualized by rendering techniques.

The algorithm was implemented in a program running under the Linux operating system. The graphic user interface was written in Java, 


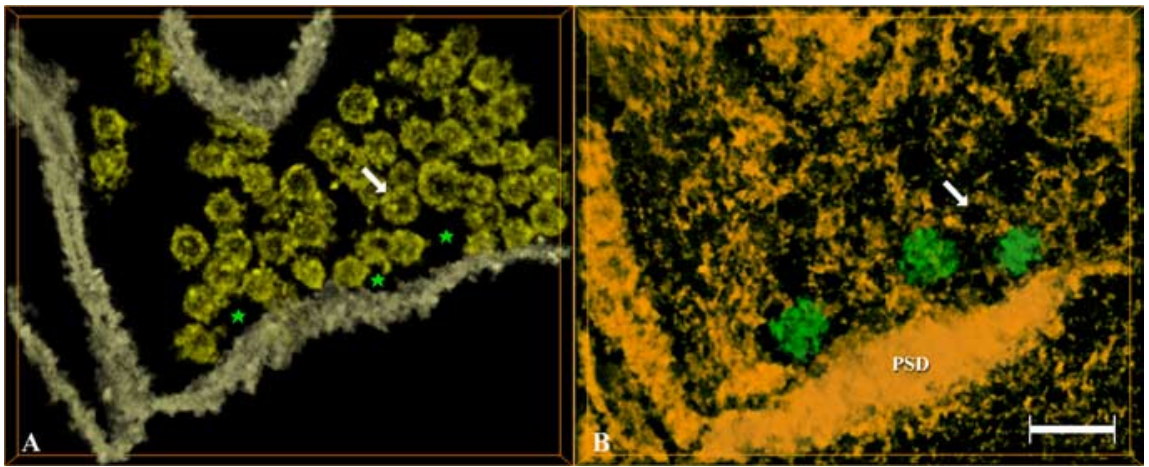

Figure 3. Semiautomatic segmentation of the reconstructed synapse. To understand the relationships that vesicles and plasma membranes establish with the cytomatrix, the two elements were split and presented in "complementary" maps. $\boldsymbol{A}$ shows the membrane map. Vesicles appear as spherical bodies, and plasma membranes appear as wavy planes highlighting the boundaries of the terminals. The unit membrane pattern is observed in the small region (left side) that was oriented perpendicular to the plane of the membrane. At the active zone, vesicles are arranged in arches, circumscribing empty spaces, indicated by green stars. At the cytoplasm, the vesicles exhibited a more random orientation, although, at places, they appear to be arranged in single-file rows. $\boldsymbol{B}$ shows the cytomatrix map. At the active zone, large particles representing the syndesomes comprised the cytomatrix (green). These structures occupy the empty center of the coronas of vesicles seen in $\boldsymbol{A}$ (green stars). The surface of the syndesomes contains interruptions indicative of their cage-like structure. Note that, in the membrane maps, the middle syndesome was obscured by three vesicles in "front" of it. Note also the presence of the syndesome on the right, unresolved in the plane section of this synapse in Figure 1. The white arrows in both panels indicate a vesicle and the void created in the cytomatrix by the segmentation. Scale bar, $95 \mathrm{~nm}$.

\section{Table 2. Quantifications of neocortical synapses}

\begin{tabular}{ll}
\hline Presynaptic terminal & \\
Length of active zone & $7.6 \mu \mathrm{m}$ \\
Area of active zone & $0.45 \mu \mathrm{m}^{2}$ \\
Syndesome & 20 \\
Number & $1.0 \pm 0.08(n=28)$ \\
x/y ratio & $59 \pm 4.5(n=58)$ \\
Diameter (nm) & $106(52 \%) ; 162$ \\
Center-to-center spacing (nm) & $(35 \%) ; 232(13 \%)$ \\
& 44 \\
Density ( $\mu m^{2}$ of active zone) & 7 \\
Number of attached vesicles & \\
Synaptic vesicles & 912 \\
Total number of vesicles & $18 \%$ \\
Total number of vesicles attached to syndesomes & $12 \%$ \\
Vesicles attached to syndesome only (second tier) & $7 \%$ \\
Vesicles attached to syndesome and active zone (first tier) & $4 \%$ \\
Vesicles attached to syndesomes and hemifused & $57 \%$ \\
First tier vesicles that were hemifused & $1 \%$ \\
Hemifused vesicles attached to two syndesomes & $0.5 \%$ \\
Fully fused vesicles &
\end{tabular}

The table summarizes data collected from 17 reconstructions of rat neocortical synapses. The number of syndesomes (20) and vesicles (912) refer to "complete" organelles after correcting for those that are incomplete and located at the edges of the sections.

whereas all intensive computational routines were written in "C." Display of images and volumes also used NIH ImageJ (Abramoff et al., 2004) and Bsoft (Heyman, 2001) software packages.

Resolution of the maps. We estimated the resolution of the maps by Fourier shell correlation (FSC) (Van Heel, 1987) and from the thickness of the phospholipids bilayer (Zampighi et al., 2005). After their refinement with global and local strategies, the resolution was estimated from the dimensions of the "unit membrane" pattern (Robertson, 1959, 1987; Zampighi et al., 2005). Because FSC requires two independent 3D reconstructions, we split the series into subsets comprising odd and even numbered images and used them to reconstruct two independent 3D maps. The reconstructed volumes were partitioned in the subvolumes as defined previously, and each one was reconstructed two times using either even or odd projections of the original series and then compared with FSC to measure local resolution. Because each reconstruction was calcu- lated from one-half the dataset, FSC yielded undervalued estimates of the resolution in the maps. A more realistic estimate was obtained using the equation described by Cardone et al. (2005), which is the correlation curve defined by the following equation: even/odd FSC $=2 \mathrm{FSC} /$ $($ FSC +1$)$. In this case, we estimated the resolution by cutting the curve at either 0.5 or 0.3 .

The thickness of the unit membrane pattern was measured in individual planes selected from three refined 3D maps. For each map, we selected five planes, and, in each plane, we performed 10-15 measurements using the measuring editor of the Amira package. Because there was significant variability in the distribution of the densities comprising the pattern, we measured regions of the plasma and vesicular membrane in which the dense layers appeared equivalent. In these regions, we measured the distance between the centers of the layers. This method eliminated the bias in selecting the boundaries of the dense layers. By comparing the resolution between both methods, we have estimated that the resolution of the conical maps was $\sim 3 \mathrm{~nm}$ (Zampighi et al., 2005, 2006).

Quantifications. In 17 tomograms, we quantified (1) the length and area of the active zone, (2) the number and diameter of the syndesomes as well as their center-to-center spacing, and (3) the vesicles in the terminal, including the total number, those attached to the syndesome, and those both hemifused and fully fused with the active zone.

For the active zone, the length was equal to the length of the layer of density associated to the abutting postsynaptic plasma membrane measured using the measuring tool in the Amira software package. In synapses in which the active zone was highly curved, the estimates used a series of straight lines created with the Amira measuring tool to approximate this curve. The area of the active zone was estimated by multiplying the length by the distance along the $z$-axis. This distance was estimated by multiplying the number of planes containing active zone by the thickness of each plane $(0.82 \mathrm{~nm})$. In reconstructions in which the angle between the plasma membrane and the plane sections different from $90^{\circ}$, the distance along $z$ was corrected by dividing by the cosine of the angle. This increased the total area of the active zone by $\sim 15 \%$.

The number of syndesomes was tabulated using the landmarks in Amira. Measuring the diameter of the syndesome required first orienting the reconstruction so that the cage was perpendicular to the plasma membrane. In this orientation, the diameter was measured both perpendicular and parallel to the plane of the active zone. Because of the thickness of the thin section, syndesomes were often incomplete in the $z$-direction. To calculate the number of "complete" syndesomes, it was necessary to apply a correction factor to the total number of syndesomes tabulated in our reconstructions. To obtain this factor, we estimated the average thickness in the $z$-direction per syndesome $(\sim 33 \mathrm{~nm})$. Dividing this by the average syndesome diameter calculated above $(\sim 60 \mathrm{~nm})$, we arrived at the correction factor, 0.55 . This was then applied to the tabulated number of incomplete syndesome (36), yielding a total of 20 complete syndesomes. The center-to-center spacing was also measured in reconstructions containing two or more syndesomes using also the Amira measuring tool. The measurements were first binned and then plotted with the program fityk (http://www.unipress.waw.pl/fityk/). The $x$-axis represents the spacing between cages in nanometers and the $y$-axis the number of measurements after binning. The three Gaussian peaks representing the measurements were refined until reaching convergence. The parameters of the Gaussians (center, height, and width) provided the area under the curve from which the size of the subpopulations was obtained and expressed as percentage of the total area under the Gaussians.

For the quantification of the number of synaptic vesicles, landmarks from the Amira software package were created for each vesicle, the center 


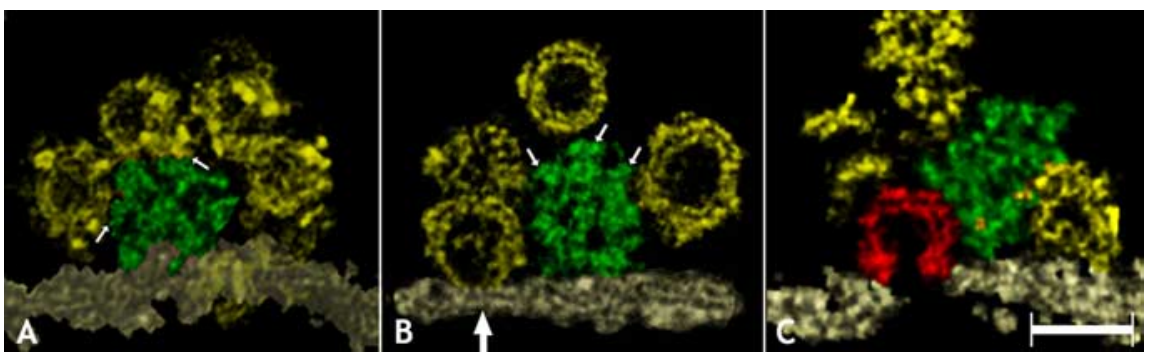

Figure 4. Segmentation of a syndesome and its corona of vesicles. This figure illustrates the relationships between the syndesome, its corona of vesicles, and the plasma membrane. $\boldsymbol{A}$ shows the syndesome comprising the central particle connected to an outer ring by radial spokes. The corona, comprising five synaptic vesicles, is intimately associated with the external ring. $\boldsymbol{B}$ shows a view oriented perpendicular to the plasma membrane, as determined by the unit membrane structure of the plasma membrane and three of the vesicles. The cage exhibits three faces and a series of spikes linking the vesicles of the corona to its surface (small arrows). The vesicle indicated by the white arrow is hemifused to the plasma membrane. C shows a fully fused vesicle (red) associated to the syndesome (green). The corona of vesicles appears incomplete and some are difficult to appreciate because the viewing angle emphasizes the opening with the plasma membrane. Scale bar, $60 \mathrm{~nm}$.

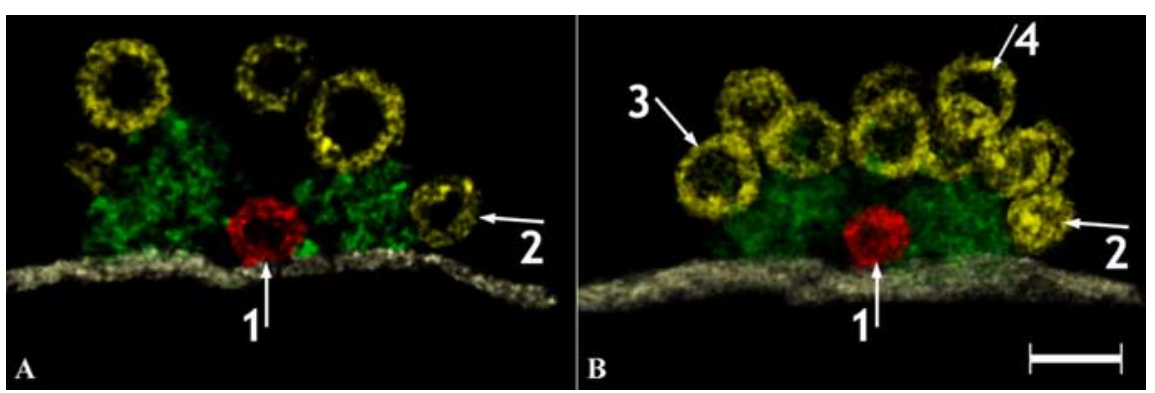

Figure 5. Hemifused vesicles associated with two cages. $\boldsymbol{A}$ is a plane of a small region of the active zone in which a synaptic vesicle (red) is attached to two cages (green). $\boldsymbol{B}$ is a portion of the rendered volume of the same region. Four classes of vesicles were labeled with arrows: (1) those attached to two cages and the active zone (arrow 1); (2) those attached to one syndesome and the active zone (first tier; arrow 2); (3) those attached to a syndesome but separated from the plasma membrane (second tier; arrow 3); and (4) those attached to filaments of the cytomatrix (arrow 4). Scale bar, $60 \mathrm{~nm}$.

of which was located within the reconstruction; these markers were then counted. After tabulating the total number of vesicles in the terminal, we counted the vesicles of the coronas around syndesomes. These vesicles were classified into two types that were counted separately: (1) those attached to the syndesome and the active zone (first tier), and (2) those attached only to the syndesomes and $\sim 50 \mathrm{~nm}$ away from the plasma membrane ("second tier"). First-tier vesicles were further subdivided into (1) those in contact with the plasma membrane, (2) those that merged their external leaflets with the plasma membrane (hemifused), and (3) those that were fully fused with the plasma membrane ("omega" figures). The numbers of these three types of first-tier vesicles were expressed as percentages of the total number in the terminal (Table 2).

Comparison with a clathrin model. We compared the structure of the polyhedral cages with the faces of the clathrin cage model available in the Protein Structure Data Bank (PDB) for the hexagonal barrel structure, reconstructed from cryomicroscopy data (Fotin et al., 2004). The atomic models of both the independent part and the full cage have been published (models 1XI4.pdb and 1XI4.pdb1, respectively). To focus our attention on the polygonal faces, we cut the distal segment of the chains and the terminal domains, which remain inside the cage. Then we used the program SITUS (Wriggers et al., 1999) to create a density map of the structure at the same pixel size as our reconstruction. To solve the problem of entire cages being larger than the thickness of the reconstructed volume, we compared only the regions made by two pentagons and one hexagon. These regions of the model were extracted from the PDB files and used to create the corresponding density maps. We then manually positioned the model in our maps and checked that the overall size of the edges and the value of the angles were compatible.

\section{Results}

\section{Overall structure}

Rat neocortical synapses are asymmetric junctions formed by the association of presynaptic and postsynaptic neurons (Fig. $1 A$ ). As in studies based on the analysis of single projections, their defining characteristics are clusters of vesicles in the presynaptic neuron, a layer of dense material on the plasma membrane of the postsynaptic neuron [postsynaptic density (PSD)] (Figs. $1 A, 2)$ and the intervening extracellular space separating both neurons (the synaptic cleft). In neocortical synapses, the width of the cleft was approximately twice that of the extracellular space of nonsynaptic regions ( $\sim 45$ vs $15-20 \mathrm{~nm})$. In contrast, the width of the extracellular cleft of rat hippocampal CA1 synapses is smaller, and the active zone exhibits the pentalamellar structure reminiscent of electrical synapses (Skisou et al., 2007).

The length of the postsynaptic density defines the active zone, the region in which vesicles fuse with the plasma membrane of the presynaptic neuron. The cytoplasm of the presynaptic neuron also contains cytoskeletal elements (the cytomatrix), the structure of which depends on their location in the terminal. At the active zone, the cytomatrix features dense particles, called here syndesomes (from Greek, syndeo, to associate, attach, or connect, and soma meaning body). Deeper within the cytoplasm, however, the cytomatrix comprised long filaments and smaller particles (Fig. $1 A$, red arrows). These synaptic organelles were observed in planes sectioned along the $x, y$, and $z$ directions (Fig. $1 A-C$ ), as well as by viewing the maps with volume-rendering methods (Fig. 2).

\section{The active zone}

Having shown that the reconstructed volume of the synapse exhibited the consensus structure deduced from projections (Palay and Palade, 1955; Gray, 1963; Peters et al., 1991), we proceeded to analyze the active zone in 17 conical reconstructions (for a total length of $\sim 7.6 \mu \mathrm{m}$ and area of $\sim 0.45 \mu \mathrm{m}^{2}$ ). Analysis of these regions throughout the reconstructions indicated the presence of complexes comprising a centrally placed syndesome surrounded by a corona of synaptic vesicles (Figs. $1 \mathrm{~A}, 2$, yellow and green). We estimated the density of syndesomes to be $\sim 44 / \mu \mathrm{m}^{2}$, with $\sim 18 \%$ of the total number of vesicles in the terminal ( 164 of 912 ) contained within the corona. The vesicles of the corona were distributed along the surface of the syndesome, appearing complete only when viewed by volume-rendering methods (compare Figs. 1 A, 2). The syndesome-vesicle complex was present in 16 of the 17 reconstructions collected for the study. In none of the reconstructions, however, did syndesomes include vesicles in their lumens.

With semiautomatic segmentation (Salvi et al., 2008), the process of additional analysis was made dynamic, allowing enhanced control over individual organelles and their constituent parts. Colors demarcating known regions of the cell could be easily 


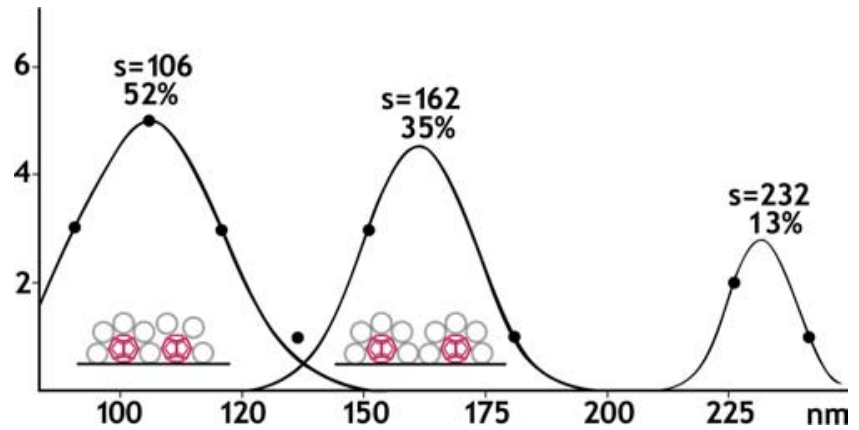

Figure 6. Center-to-center spacing of neighboring polyhedral cages. The values of the center-to-center were first binned and then plotted with the program fityk (http://www. unipress.waw.pl/fityk/). The $x$-axis represents the spacing between cages in nanometers, and the $y$-axis represents the number of measurements after binning. Visual inspection indicated the presence of three peaks that were refined until reaching convergence. The parameters of the Gaussian curves (center, height, and width) provided the area under the curve from which the size of the different subpopulations was obtained and expressed as percentage of the total area under the peaks. The two peaks from the left are centered at 106 and $162 \mathrm{~nm}$ and represent, respectively, 52 and $32 \%$ of the total area. Thus, $52 \%$ of the area of the active zone contains syndesomes separated by a single vesicle, whereas $32 \%$ contains syndesomes separated by two vesicles (indicated by the schematic drawings under the respective curves). The predominance of measurements in these bins suggests significant hexagonal close packing in the arrangement of units on the active zone. The remaining small peak is centered at $232 \mathrm{~nm}$ and represents $13 \%$ of the total area, suggesting that regions that deviate from perfect hexagonal close packing also occur in the active zone (see Fig. 9).

added or changed, and the regions themselves could be made translucent or temporarily removed. A combination of these approaches yielded several strategies. First, we segmented the vesicles and plasma membranes separately from the syndesomes and other components of the cytoskeleton. We called these complementary maps the membrane and the cytomatrix map (Fig. $3 A, B$, yellow, white, green, and brown). From the analysis of these complementary maps, we deduced that the cytomatrix determined the arrangement of vesicles in the presynaptic terminal. At the active zone, the membrane maps depicted vesicles as hollow spheres arranged as arches around empty spaces (Fig. 3A, stars). In the cytomatrix maps, spherical voids created by the absence of vesicles surrounded particles of distinct dimensions and shape representing the syndesomes (Fig. 3B, green). Deeper within the cytoplasm, syndesomes were replaced with webs of filaments and small particles encasing vesicles and arranging them in single-file rows (Fig. $3 A, B$, arrows) (see Fig. 9B, C).

We then segmented the syndesome, the synaptic vesicles, the plasma membrane, and the filaments of the cytoskeleton assigning a different color to each element (see Figs. 4, 5, 7, 8). Using this strategy, we found that short spikes linked vesicles of the corona to the surface of the syndesomes (Fig. $4 A, B$, arrows). We also found that, whereas vesicles of the corona represented $\sim 18 \%$ of the total, only those at their base were abutting the active zone. These vesicles represent $\sim 7 \%$ of the total (64 of 912) and are referred to as first tier because of their proximity to the plasma membrane.

We then studied the region of contact that first-tier vesicles establish with the plasma membrane to determine whether it comprised one or two membranes. We found that $\sim 57 \%$ of firsttier vesicles (36 of 64$)$ established a small (20-25 nm diameter) region of contact in which their external leaflets merged with the plasma membrane (hemifusion). Hemifused vesicles associated with syndesomes represent $\sim 4 \%$ of the total (36 of 912), strengthening previous studies indicating that the overwhelming majority of synaptic vesicles contiguous with the active zone are
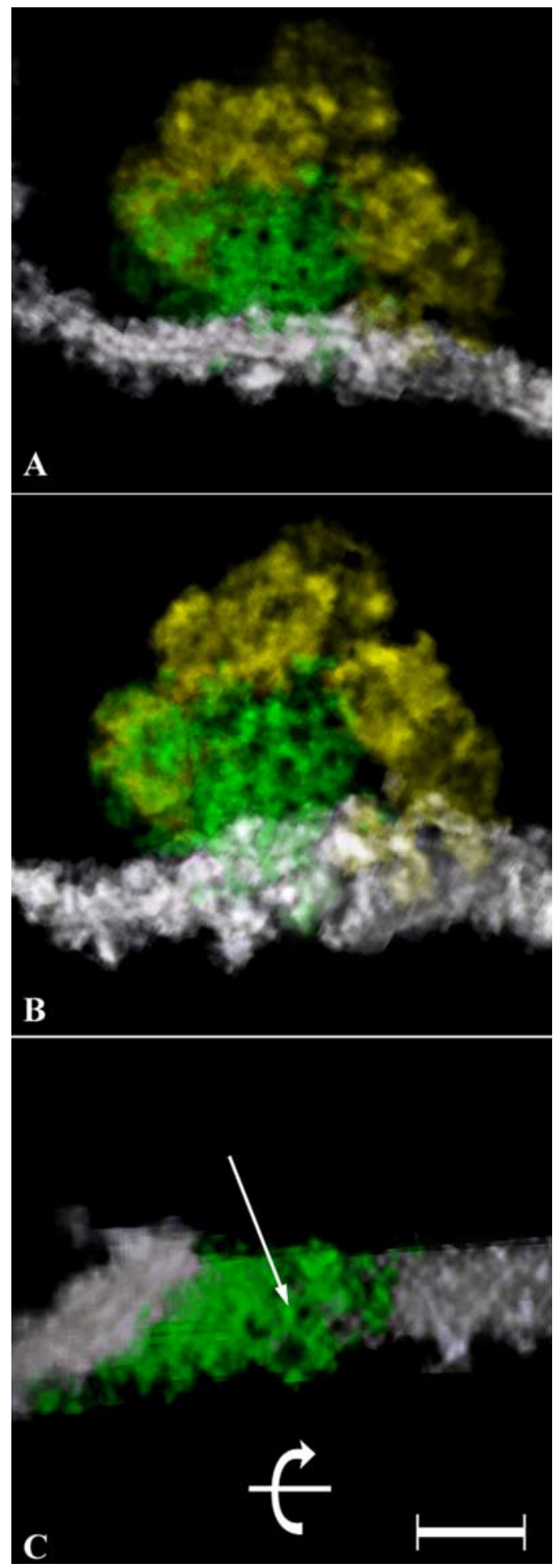

Figure 7. Syndesomes are polyhedral cages. A small region of the active zone (white band), a syndesome (green) and its corona of synaptic vesicles (yellow), was segmented and rotated around an axis represented by the plane of the plasma membrane. $\boldsymbol{A}$ shows a view oriented perpendicular to the active zone, as determined by the trilayer structure of the plasma membrane (white band). The syndesome appears as a cage with polygons, discernable by the four dark regions of their centers, arranged in a row. $\boldsymbol{B}$ shows a view rotated $\sim 30^{\circ}$ from the perpendicular plane, as determined from the disappearance of the trilayer structure. A larger region of the surface of the cage is revealed demonstrating the series of polygons tessellating its surface. ( shows the en face view of the plasma membrane $\left(\sim 0^{\circ}\right)$. This view shows the entire thickness of the section. Only a part of the cage comprising three polygons meeting at a vertex is clearly visible (white arrow). To view the surface of the polyhedral cage, the vesicles of the corona were excluded. The straight line and the arrow show the direction of the rotation used to generate the views. Scale bar, $90 \mathrm{~nm}$. 

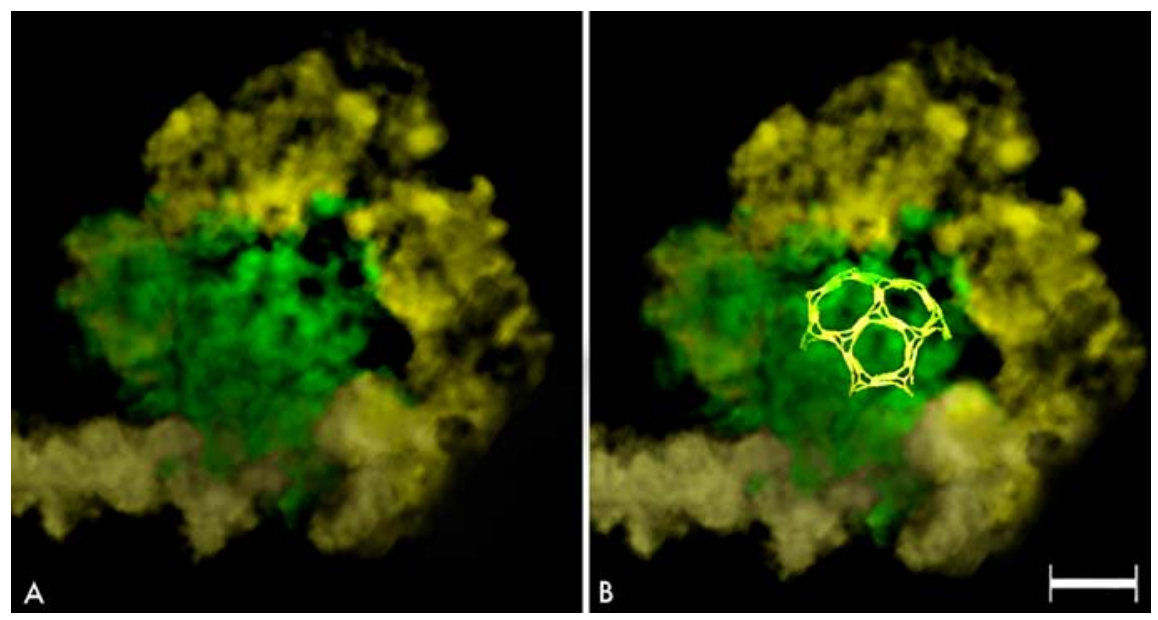

Figure 8. Comparison with a clathrin model. We compared the structure of the polyhedral cages $(\boldsymbol{A})$ with the faces of the clathrin cage model available in the Protein Structure Data Bank for the hexagonal barrel structure, reconstructed from cryomicroscopy data by averaging thousands of cages and by applying D6 symmetry (1XI4.pdb and 1XI4.pdb1) (Fotin et al., 2004). In the model of the hexagonal barrel, there are two types of hexagons: those lining the top (or the bottom) of the barrel, which is surrounded only by pentagons, and the hexagons of the central belt, which are in contact with both pentagons and hexagons. $\boldsymbol{B}$, The hexagon coming from the belt produced a slightly better fit. Although it was not possible to observe a precise correspondence between the two lattices, both were comparable in size and shape. Scale bar, $60 \mathrm{~nm}$.

hemifused (Zampighi et al., 2006). In contrast, vesicles fully fused with the active zone (Fig. $4 C$, the omega figures) represent only $\sim 0.4 \%$ of the total number of vesicles in the terminal.

We also examined the vesicles between neighboring syndesomes. We observed that often a single synaptic vesicle spanned the space between two syndesomes (Fig. $5 A, B$, red). Moreover, they were hemifused with the plasma membrane, consisting of $\sim 1 \%$ of the total number of vesicles in the terminal ( 10 of 912 ). We also found situations in which two vesicles occupied the space between neighboring syndesomes (see center-to-center measurements). Regions in which units of syndesomes with their surrounding corona of vesicles were not contiguous were rare, representing $\sim 13 \%$ (Fig. 6 ). Syndesomes and their associated vesicles thus occupied the great majority of the area of the active zone, leaving few empty spaces for unassociated vesicles to contact the plasma membrane.

From the quantifications in Table 2, synaptic vesicles could be classified as those associated with syndesomes and arranged in coronas $(\sim 18 \%$ of total), those associated simultaneously with syndesomes and the active zone ( $\sim 7 \%$ of total), those that are hemifused ( $\sim 4 \%$ of total), those associated to the active zone and two syndesomes ( $1 \%$ of total), and those fully fused $(\sim 0.4 \%)$ to the plasma membrane.

\section{Syndesomes are polyhedral cages}

An unexpected conclusion from semiautomatic segmentation and analysis of the active zone was that syndesomes were polyhedral cages (Figs. $3 B, 4 A$, green). The first indication arose from the analysis of planes. They revealed a central particle connected to an external ring (Fig. $4 A, B$ ) and short spokes connecting the cages to the vesicle of the corona (Fig. $4 B, C$ ). The cages were approximately spherical, with an $x / y$ ratio of $1.0 \pm 0.08$ (mean \pm SD; $n=29$ ), where $x$ and $y$ are the dimensions parallel and perpendicular to the plasma membrane. The diameter of the cage measured $59 \pm 4.5 \mathrm{~nm}$ (mean $\pm \mathrm{SD} ; n=58)$. To obtain accurate values for the center-to-center spacing, the measurements were fitted with three Gaussian curves, the peaks of which measured $106 \mathrm{~nm}$ ( 52\%), $162 \mathrm{~nm}(35 \%)$, and $232 \mathrm{~nm}$ (13\%) (Fig. 6). The peak at $106 \mathrm{~nm}$ represents the packing of units of polyhedral cages with their corresponding coronas of vesicles such that they overlap, sharing a vesicle between them. The peak at $162 \mathrm{~nm}$ represents packing of two abutting units, and the smaller peak at $232 \mathrm{~nm}$ represents the areas of active zone not filled by units of syndesomes and associated vesicles, demonstrating as expected that the arrangement of units on the plane deviated from perfect hexagonal close packing.

Rotating the polyhedral cage and its corona of vesicles provided additional information about the polyhedral structure of the syndesome (Fig. $7 A-C$ ). We used the appearance of the unit membrane pattern to determine that the views spanned $90^{\circ}$ rotations. Views oriented either perpendicular or rotated $\sim 30^{\circ}$ with respect to the active zone revealed the characteristic arrangement of rings tessellating the surface (Fig. $7 A-C$ ). In contrast, en face views revealed the active zone as a plane and exposed the rings of the polyhedron in contact with the corona of vesicles (Fig. 7C). In these views, the distance between vertices of the cage measured 35-38 nm (Fig. 7C).

The overall structure of the syndesome somewhat resembles that of the clathrin cage comprising "coated" vesicles, the organelles involved in membrane retrieval in the presynaptic terminal (Slepnev and De Camilli, 2000). Consequently, we compared the cage in the syndesome with the clathrin lattice of the hexagonal barrel structure, reconstructed from cryomicroscopy data (models 1XI4.pdb and 1XI4.pdb1) (Fotin et al., 2004). In the model of the hexagonal barrel, there are two types of hexagons: those lining the top (or the bottom) of the barrel, which is surrounded only by pentagons, and the hexagons of the central belt, which are in contact with both pentagons and hexagons. Maps from each substructure were fitted into the cage of the syndesome. Within these two regions, it appeared that the hexagon coming from the belt produced a better fit. Although it was not possible to observe a precise correspondence between the two lattices, both were comparable in shape (Fig. $8 A, B)$. The length of the clathrin leg in the syndesome, however, was somehow shorter than in the asymmetric unit of the molecular model. This difference might result from the shrinkage of the section induced by radiation damage during the collection of the series (Zampighi et al., 2005). The cages were irregular and did not correspond to any of the perfect polyhedron described in the literature. However, the mean diameter of the syndesome was in good agreement with the diameter of the mini-coat clathrin structure $(62 \mathrm{~nm})$ described by Fotin et al. (2004).

Finally, we also examined the cytomatrix associated with vesicles deeper within the terminal. In this region, filaments of variable length comprised the cytomatrix (Fig. 9A-C). Short filaments formed webs around vesicles (Fig. 9B, $C$, white arrow). Long filaments tethered neighboring synaptic vesicles to the active zone and interconnected neighboring cages (Fig. $9 A-C)$. The diameter of these filaments was thinner than the short filaments interconnecting vesicles in presynaptic terminals of the rat hippocampal CA1 area ( $~ 6$ vs $10-15 \mathrm{~nm})$ (Siksou et al., 2007). 


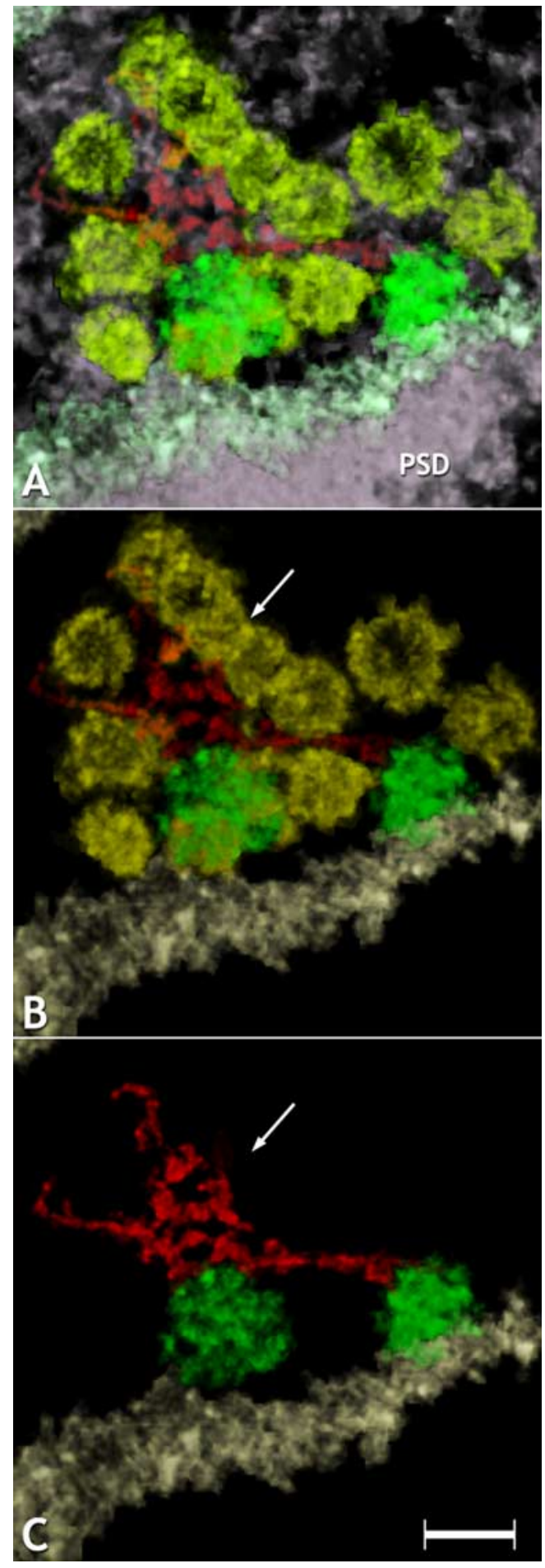

Figure 9. Relationships between cages, vesicles, and the cytomatrix. This figure illustrates the interactions between polyhedral cages (green), the vesicles (yellow), and the filaments (red) in the cytoplasm of the terminal. This region seen here was cropped from the map in Figure 3. $\boldsymbol{A}$ shows two cages (green) associated to the active zone and surrounded by vesicles (yellow). The densities occupying the spaces between these organelles contain components of the cytoskeleton (the cytomatrix). These on top of the cages were colored red for emphasis. PSD indicates the layer of density associated to the postsynaptic membrane. $\boldsymbol{B}$ shows the same region after removing the cytoskeleton, including PSD. The filaments colored red attached to the syndesomes were maintained to demonstrate the location of vesicles within the terminal. $\boldsymbol{C}$ shows the region after removing the synaptic vesicles. This view shows the syndesomes associated to the plasma membrane and the interconnecting filaments. Vesicles were attached along the entire length of filament. At places, the cytoskeleton appeared as islands in which filaments formed a web decorated with small particles. Arrows indicate the relationship of vesicles to cytomatrix. Scale bar, $60 \mathrm{~nm}$.
Table 3. The average synapse

\begin{tabular}{lc}
\hline Diameter $(\mu \mathrm{m})$ & 0.5 \\
Area $\left(\mu \mathrm{m}^{2}\right)$ & 0.196 \\
Number of vesicles & 397 \\
Number of syndesomes & $8-9$ \\
Vesicles in corona & 7 \\
First-tier vesicles & 28 \\
Hemifused vesicles & 16 \\
Hemifused attached to two syndesomes & 4
\end{tabular}

The information from Table 2, normalized to fit an average synapse. These data were used to construct the model of the synapse in Figure 10.

\section{Discussion}

The principal conclusion of our study is that a variable number of units, or complexes, each comprised a polyhedral cage, or syndesome, with a surrounding corona of vesicles that constitute the active zone of rat neocortical synapses. Although the polyhedral structure of this cage is first noted here, the existence of these units is supported by a wealth of published information, indicating their presence in a variety of central synapses (Gray, 1963; Ackert et al., 1972; Pfenninger et al., 1972; Peters et al., 1991; Zampighi and Fisher, 1997). Furthermore, a possible role of the polyhedral cage in docking and fusion is suggested by the strong presence of vesicles in their corona that are hemifused or fully fused to the plasma membrane (Fig. $4 B, C$ ).

The particular coronal arrangement of vesicles around the perimeter of the syndesome and their absence from the lumen were initial indications that syndesomes might function as "docking stations" (Figs. 1-3A). This arrangement became evident only after vesicles and the presynaptic cytomatrix were segmented and reconstructed in separate maps (Fig. $3 A, B$, yellow, green, and brown). Estimates of the number of vesicles comprising these coronas (Table 2) indicated that essentially all vesicles at the active zone were associated to at least one syndesome. To test this conclusion, we examined the 3D structure that different vesicles of the corona establish with the plasma membrane at the active zone.

Arrangement in coronas defined two types of vesicles: those at their base (first tier) and those at their crook, located $\sim 50 \mathrm{~nm}$ from the plasma membrane (second tier). As reported in previous studies, approximately one-half of the vesicles occupying the first tier position were hemifused with the plasma membrane (Zampighi et al., 2006). Here, we report the unexpected observation that vesicles hemifused with the active zone are also associated with polyhedral cages (Fig. $4 B, C$ ). Based on the relationship of hemifused and fully fused vesicles with syndesomes (Fig. $4 B, C$ ), we thus suggest that the binding of vesicles to polyhedral cages is a constitutive part of the process of vesicle docking to the active zone.

To put the quantifications performed in this study (Table 2) into context, the number of units were normalized to fit an average presynaptic terminal of spherical shape (Table 3). Each unit comprised a syndesome and its contiguous corona of synaptic vesicles (Fig. 10 A, here shown with connections into the remainder of the terminal), and resultant spherical shapes had diameters equal to the mean length of the active zone in the 17 reconstructions, $0.5 \mu \mathrm{m}$ (Table 3). Extrapolating from our data, we calculated that the average terminal should have approximately eight to nine units (Table 3), arranged along the plane of the active zone.

Based on the density of polyhedral cages $\left(\sim 44 / \mu \mathrm{m}^{2}\right)$ and first-tier vesicles $\left(\sim 140 / \mu \mathrm{m}^{2}\right)$ as well as the presence of vesicles occupying the spaces between neighboring cages (Fig. 5), we pro- 

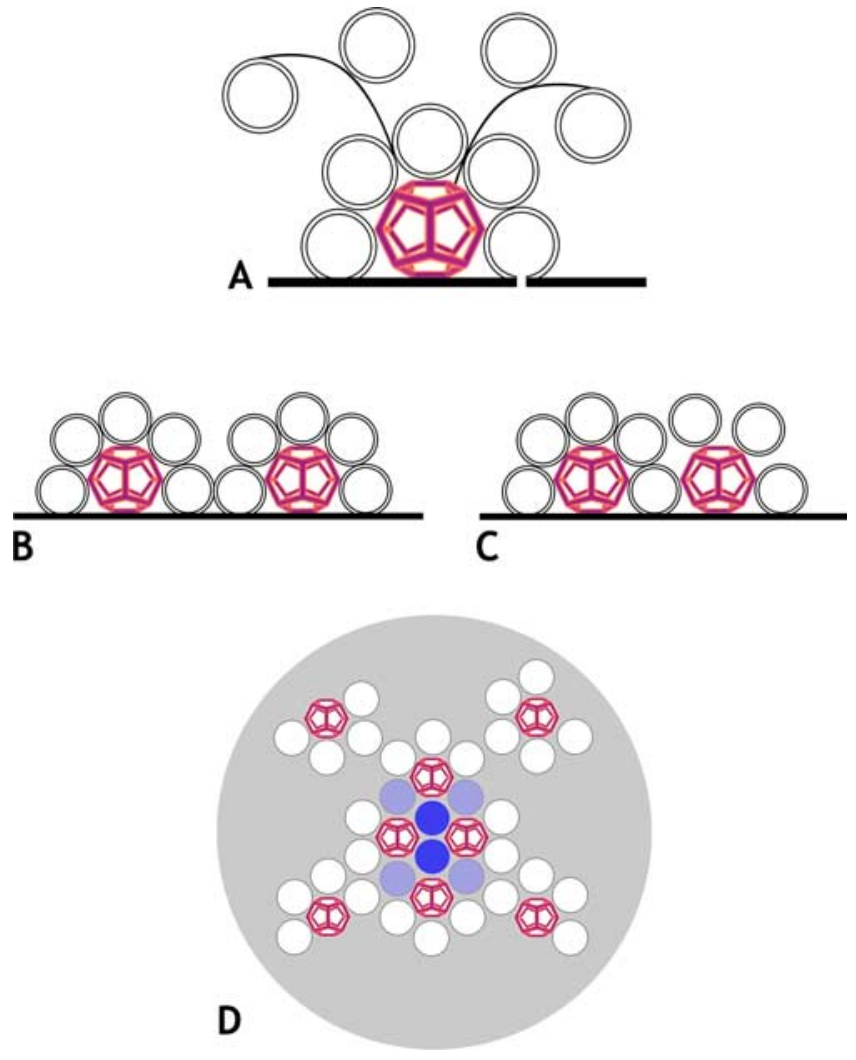

Figure 10. Model of an average synapse. The model predicts that the terminals of neocortical synapses are constructed of units comprising a polyhedral cage surrounded by a corona of vesicles. $\boldsymbol{A}$ depicts a single unit, represented with one vesicle at the base of the corona hemifused and one fully fused. Vesicles deeper within the terminal are shown tethered to the active zone via long filaments. In theory, a single synaptic unit could form a release site, although the existence of a terminal of this size was not determined in this study. $\boldsymbol{B}$ and $\boldsymbol{C}$ show neighboring units associated laterally by either one (106 nm spacing) or two ( $162 \mathrm{~nm}$ spacing) vesicles. Both types of lateral association between synaptic units were observed in the reconstructions (Figs. 3, 7). The predominance of cages separated by a single vesicle $(\boldsymbol{C})$ gives rise to the prediction that synaptic units at the active zone exhibit hexagonal closed packing. $\boldsymbol{D}$ depicts the possible arrangement of eight synaptic units on the plane of the active zone. For simplicity, four units are separated by one intervening vesicle between cages (i.e., close packing) and four by two vesicles. Note that, at the close packed region, the vesicles are linked to two or three cages (dark and light circles). We speculate that the association with multiple cages extends the length of time these vesicles remain flush against the plasma membrane, thus increasing the probability for docking and fusion. Therefore, the model predicts that the packing of these units determines the number of vesicles available for immediate release.

pose that a significant degree of hexagonal close packing must occur between units on the plane of the active zone, conforming to the two arrangements shown in Figure 10, $A$ and $B$. A consequence of close packing is that a number of vesicles would be linked to two and even three polyhedral cages (Fig. 10D, dark and light blue circles). We speculate that association with multiple cages extends the length of time these vesicles remain flush against the plasma membrane, thus increasing the probability for docking and fusion. Nevertheless, hexagonal close packing itself does not affect the result that very little docking can occur unassociated from polyhedral cages, revealed by our quantifications of the density of cages and first-tier vesicles.

The possibility that syndesomes play a role in vesicle docking was anticipated by the analysis of isolated fractions of active zones that contained syndesomes, called "grid particles" (Phillips et al., 2001). These studies identified $\sim 12$ proteins belonging to different families [cytoskeletal (spectrin, tubulin, and actin), cell adhesion (N-cadherin, calcium/calmodulin-dependent serine kinase, and $\beta$-catenin), endocytosis (clathrin, dynamin), vesicle fusion (N-ethylmaleimide-sensitive fusion protein [NSF] and Munc18) and neurotransmitter loading (V-ATP synthetase)]. Although we cannot, as of yet, specifically identify individual proteins in conical maps, the 3D structure of syndesomes reflects the properties that have been attributed to some of these proteins in the literature. For example, cytoskeletal proteins, such as actin, may represent the long filaments tethering syndesomes to undocked vesicles (Doussau and Augustine, 2000) (Fig. 9A-C). Additionally, the presence of vesicle fusion proteins, such as NSF and Munc-18, both thought to function in combination with SNAREs (Rizo and Sudhof, 2002), may help to explain the link between syndesomes and SNAREs and thereby account for the hemifused and fully fused vesicles in the coronas.

The identification of clathrin in the fractions containing syndesomes (Phillips et al., 2001), however, was perplexing, because this protein is not traditionally associated with exocytosis. To explore this possibility, we fitted two kinds of hexagonal faces, reconstructed from cryomicroscopy of the barrel cage (Fotin et al., 2004), into our reconstruction (Fig. $8 A, B$ ). The congruency of the angles and sides of the polyhedra confirmed the possibility that the structure of the syndesome as a polyhedral cage was likely attributable to clathrin. However, additional investigation is required.

Syndesomes are not the only protein complexes that have been identified in the presynaptic terminal with putative roles in vesicle tethering, docking, and priming (for review, see Zhen and Jin, 2004). Among the others are (1) the tripartite complex comprising $\alpha$-Rab3-interacting molecule/Munc13/Rab3, that is thought to bring vesicles in contact with the priming machinery (Dulubova et al., 2005), (3) the Munc18/syntaxin 1 complex that is thought to prime vesicles to a form-competent state (Rizo and Sudhof, 2002), and (3) the large Piccolo and Bassoon proteins that act as platforms for a myriad of other proteins and tether vesicles to the active zone (Fenster et al., 2000). Although it is likely that these other proteins interact with syndesomes, there is currently a lack of structural information regarding the location of these complexes. Until this improves, especially with respect to hemifused or fully fused vesicles, any attempt to unravel the possible relationships these complexes might have with syndesomes at the active zone is thwarted.

We thus conclude that the dense particles identified by Gray and others decades ago, here called syndesomes, represent novel protein complexes with distinct polyhedral structure, likely a result of clathrin. Their location exclusively at the active zone and their association with vesicles that are hemifused and fully fused with the plasma membrane strongly suggest that they function as docking stations with a role in neurotransmitter release. It seems likely that they are a critical player in organizing docked vesicles, regulating the size and configuration of the immediately releasable pool.

\section{References}

Abramoff MD, Magelhaes PJ, Ram SJ (2004) Image processing with Image J. Biophoton Int 11:36-42.

Ackert H, Pfenninger K, Moor K, Sandri C (1972) Freeze-etching and cytochemistry of vesicles and membrane complexes in synapses of the central nervous system. In: Structure and function of synapses (Pappas GD, Purpura DP, eds), pp 67-86. New York: Raven.

Bloom FE, Aghajanian GK (1966) Cytochemistry of synapses: a selective staining method for electron microscopy. Science 154:1575-1577.

Cantele F, Zampighi L, Radermacher M, Zampighi G, Lanzavecchia S (2007) Local refinement: an attempt to correct for shrinkage and distortion in electron tomography. J Struct Biol 158:59-70. 
Cardone G, Grunewald K, Steven AC (2005) A resolution criterion for electron tomography based on cross-validation. J Struct Biol 151:117-129.

Couteaux R, Pecot-Dechavassine M (1970) Synaptic vesicles and pouches at the level of the "active zones" of the neuromuscular junction. C R Acad Sci Hebd Seances Acad Sci D 271:2346-2349.

Dick O, Dieck S, Altrock WD, Ammermuller J, Gunderfilger ED, Brandstatter JH (2003) The presynaptic active zone protein bassoon is essential for photoreceptor ribbon synapse formation in the retina. Neuron 37:775-786.

Doussau F, Augustine GJ (2000) The actin cytoskeleton and neurotransmitter release: an overview. Biochimie 82:353-363.

Dulubova I, Lou X, Lu J, Huryeva I, Alam A, Schneggenburger R, Sudhof TC, Rizo J (2005) A Munc13/RIM/Rab3 tripartite complex: from priming to plasticity. Eur Mol Biol Org 24:2839-2850.

Fenster SD, Joon CW, Zhai R, Cases-Lanhoff C, Voss B, Garner AM, Kaempf U, Kindler S, Guldenfinger ED, Garner CC (2000) Piccolo, a presynaptic zinc finger protein structurally related to bassoon. Neuron 25:203-214.

Fotin A, Cheng Y, Sliz P, Grigorieff N, Harrison SC, Kirchhausen T, Waltz T (2004) Molecular model for a complete clathrin lattice from electron cryomicroscopy. Nature 432:573-579.

Gray EG (1963) The granule cells, mossy synapses and Purkinje spine synapses of the cerebellum: light and electron microscope observations. J Anat Lond 97:101-106.

Harlow ML, Ress D, Stoschek A, Marshall RM, McMahan UJ (2001) The architecture of active zone material of the frog's neuromuscular junction. Nature 409:479-484.

Heyman BJ (2001) Bsoft: image and molecular processing in electron microscopy. J Struct Biol 133:156-169.

Hirokawa N, Sobue K, Kanda K, Harada A, Yorifuji H (1989) The cytoskeletal structure of the presynaptic terminal and molecular structure of synapsin 1. J Cell Biol 108:111-126.

Jahn R, Scheller RH (2006) SNAREs-engines for membrane fusion. Nat Rev Mol Cell Biol 7:11-126.

Landis DM, Hall AK, Weinstein LA, Reese TS (1988) The organization of the cytoplasm at the presynaptic active zone of a central nervous system synapse. Neuron 1:201-209.

Lanzavecchia S, Cantele F, Bellon PL, Zampighi L, Kreman M, Wright EM, Zampighi GA (2005) Conical tomography of freeze-fracture replicas: a method for the study of integral membrane proteins inserted in phospholipid bilayers. J Struct Biol 149:87-98.

Lenzi D, Runyeon JW, Crum J, Ellisman MH, Roberts WM (1999) Synaptic vesicle populations in saccular hair cells reconstructed by electron tomography. J Neurosci 19:119-132.

Meinerzhagen IA (1996) Ultrastructure and quantification of synapses in the insect nervous system. J Neurosci Methods 69:59-73.

Meinerzhagen IA, Govind CK, Stewart BA, Carter JM, Atwood HL (1998) Regulated spacing of synapses and presynaptic active zones at larval neuromuscular junctions in different genotypes of flies Drosophila and Sarcophaga. J Comp Neurol 393:482-492.

Palay SL, Palade GE (1955) The fine structure of neurons. J Biophys Biochem Cytol 1:68-88.

Peters A, Paley SL, Wester H (1991) The fine structure of the nervous system, Chap 5, Ed 3. New York: Oxford UP.
Pfenninger K, Ackert K, Moor H, Sandri C (1972) The fine structure of freeze-fractured presynaptic membranes. J Neurocytol 1:129-149.

Phillips GR, Huang JK, Wang Y, Tanaka H, Shapiro L, Zhang W, Shan WS Arndt K, Frank M, Gawinowicz MA, Colman DR (2001) The presynaptic particle web: ultrastructure, composition, dissolution, and reconstitution. Neuron 32:63-77.

Radermacher M (1992) Weighted back projection methods. In: Electron tomography, pp 91-116. New York: Plenum.

Rizo J, Sudhof TC (2002) SNAREs and munc18 in synaptic vesicle fusion. Nat Rev Neurosci 3:641-653.

Robertson JD (1959) The ultrastructure of cell membranes and their derivatives. Biochem Soc Symp 16:3-43.

Robertson JD (1987) The early days of electron microscopy of nerve tissue and membranes. Int Rev Cytol 100:129-201.

Salvi E, Cantele F, Zampighi L, Fain N, Zampighi GA, Lanzavecchia S (2008) JUST (Java User Segmentation Tool) for semi-automatic segmentation of tomographic maps. J Struct Biol 161:287-297.

Siksou L, Rostaing P, Lechaire JP, Boudier T, Ohtsuka T, Fejtova A, Kao HT, Greengard P, Gundelfinger ED, Triller A, Marty S (2007) Threedimensional architecture of presynaptic terminal cytomatrix. J Neurosci 27:6868-6877.

Slepnev VI, De Camilli P (2000) Accessory factors in clathrin-dependent synaptic vesicle endocytosis. Nat Rev Neurosci 1:161-172.

Triller A, Korn H (1985) Activity-dependent deformations of presynaptic grids at central synapses. J Neurocytol 14:177-192.

Van Heel M (1987) Similarity measures between images. Ultramicroscopy 21:95-100.

Von Gersdorff H (2001) Synaptic ribbons: versatile signal transducers. Neuron 29:7-10.

Vincent L, Soille P (1991) Watersheds in digital spaces: an efficient algorithm based on immersion simulations. IEEE Trans Pattern Anal Mach Intell 13:583-598.

Wriggers W, Milligan RA, McCammon JA (1999) SITUS: a package for docking crystal structures into low resolution maps from electron microscopy. J Struct Biol 125:185-195.

Zampighi GA, Fisher RS (1997) Polyhedral protein cages encase synaptic vesicles and mediate their docking to the pre-synaptic membrane. J Struct Biol 119:347-359.

Zampighi GA, Corless JM, Robertson JD (1980) On gap junction structure J Cell Biol 86:190-198.

Zampighi GA, Hall JE, Ehring GR, Simon SA (1989) The structural organization and protein composition of lens fiber junctions. J Cell Biol 108:2255-2275

Zampighi GA, Zampighi L, Fain N, Wright EM, Cantele F, Lanzavecchia S (2005) Conical tomography II: a method for the study of cellular organelles in thin sections. J Struct Biol 151:263-274.

Zampighi GA, Zampighi L, Fain N, Lanzavecchia S, Simon SA, Wright EM (2006) Conical electron tomography of a chemical synapse: vesicles docked to the active zone are hemi-fused. Biophys J 91:2910-2918.

Zhai RG, Bellen HJ (2004) The architecture of the active zone in the presynaptic nerve terminal. Physiology 19:262-270.

Zhen M, Jin Y (2004) Presynaptic terminal differentiation: transport and assembly. Curr Opin Neurobiol 14:280-287. 